\title{
Long non-coding RNAs in cancer
}

\author{
GONG ZhaoJian $^{1,2,3}$, ZHANG ShanShan ${ }^{4}$, ZHANG WenLing ${ }^{2}$, HUANG HongBin ${ }^{2,5}$, LI Qiao ${ }^{2}$, \\ DENG Hao $^{1}$, MA Jian ${ }^{1,2}$, ZHOU Ming ${ }^{1,2}$, XIANG JuanJuan ${ }^{1,2}$, WU MingHua ${ }^{1,2}$, LI XiaYu ${ }^{1}$, \\ XIONG Wei $^{1,2}$, LI XiaoLing ${ }^{1,2}$, LI Yong ${ }^{6}$, ZENG ZhaoYang ${ }^{1,2^{*}}$ \& LI GuiYuan ${ }^{1,2^{*}}$ \\ ${ }^{1}$ Hunan Key Laboratory of Nonresolving Inflammation and Cancer, Disease Genome Research Center, the Third Xiangya Hospital, Central \\ South University, Changsha 410013, China; \\ ${ }^{2}$ Key Laboratory of Carcinogenesis of Ministry of Health, Key Laboratory of Carcinogenesis and Cancer Invasion of Ministry of Education, \\ Cancer Research Institute, Central South University, Changsha 410078, China; \\ ${ }^{3}$ Department of Stomatology, the Second Xiangya Hospital, Central South University, Changsha 410011, China; \\ ${ }^{4}$ Department of Stomatology, Xiangya Hospital, Central South University, Changsha 410008, China; \\ ${ }^{5}$ Key Laboratory of Information System Engineering, National University of Defense Technology, Changsha 410073, China; \\ ${ }^{6}$ Department of Biochemistry and Molecular Biology, Center for Genetics and Molecular Medicine, School of Medicine, University of \\ Louisville, Louisville, KY 40202, USA
}

Received October 28, 2012; accepted November 10, 2012

Citation: $\quad$ Gong Z J, Zhang S S, Zhang W L. Long non-coding RNAs in cancer. Sci China Life Sci, 2012, 55: 1120-1124, doi: 10.1007/s11427-012-4413-9

Long non-coding RNAs (lncRNAs) are a group of RNA transcripts that exceed $200 \mathrm{nt}$ in length, yet lack significant open reading frames (ORFs) [1-4]. In contrast to small non-coding RNAs (ncRNAs), such as microRNAs (miRNAs) [4-23], small interfering RNAs (siRNAs) [24-31] and transfer RNAs (tRNAs) [32-34], there are thousands of lncRNA genes discovered during the past three years in the human genome and most of their functions remain elusive. The long nucleotide chain of lncRNAs can either form a complex spatial structure and interact with protein factors, or provide a large segment for the concurrent binding of many molecules that collectively participate in genomic imprinting, X-chromosome silencing, chromosome modification, intranuclear transport, transcriptional activation and interference, thereby regulating cell growth, differentiation, development, senescence and death [35].

Accumulating evidence supports that lncRNAs participate in many physiological processes by modulating gene expression at the epigenetic, transcriptional and posttranscriptional levels. LncRNAs regulate gene expression at the epigenetic level through DNA methylation or demethyl-

*Corresponding author (email: zengzhaoyang@xysm.net; ligy@xysm.net) ation, RNA interference, histone modifications, chromatin remodeling, etc. [36-42]. At the transcriptional level, lncRNAs depends on the relative position and sequence features of the lncRNA and the target gene to regulate gene expression. Khps1a is an lncRNA that is transcribed near the $\mathrm{CpG}$ island of the oncogene sphingosine kinase 1 (SphK1). Through binding to $3 \mathrm{CC}(\mathrm{A} / \mathrm{T}) \mathrm{GG}$ sites in the tissue-dependent differentially methylated region (T-DMR) of SphK1, Khps1a induces the demethylation of the CpG island, resulting in increased expression of SphK1 [43]. Another example is the $3^{\prime}$ end of yeast IncRNA Srg1, which overlaps the promoter of the target gene Ser 3 and inhibits Ser3 expression by occupying the binding site for transcription initiation factors in the Ser3 promoter [44]. At the transcriptional level, some lncRNAs inhibit the transcription of target genes by binding to the promoters of target genes and forming stable DNA-RNA triplex complexes [45], while others cooperate with transcription modulators in transcriptional regulation and interfere with the formation of the transcription initiation complex to repress transcription initiation and to rapidly alter gene expression patterns [46]. Post-transcriptionally, the formation of a RNA dimer via complementary base pairing between the lncRNA and the 
target mRNA can block the binding sites of transcription factors and processing-related factors, which regulate mRNA splicing, translation and degradation [47]. Some lncRNAs bind miRNAs and competitively inhibit the interaction between miRNAs and target mRNAs to modulate gene expression. Furthermore, lncRNAs can function as structural components of larger RNA-protein complexes, modulate the activity and subcellular localization of proteins, and thus play additional specific roles [36].

Deregulated expression of lncRNAs has been found in a variety of cancers [48]. Profiling lncRNA expression in five cases of HBV-associated hepatocellular carcinoma tissue and non-cancerous tissue, Yang et al. [49] found that 174 lncRNAs were deregulated in hepatocellular carcinoma. Khaitan et al. [50] discovered that 77 lncRNAs were deregulated in the melanoma cell line WM1552C; the authors further analyzed lncRNA expression in 29 melanoma patient samples and 6 normal skin samples and identified 4 lncRNAs that were deregulated in both the WM1552C cells and the melanoma patient specimens. These data implicate IncRNAs in carcinogenesis and tumor progression. However, the molecular mechanisms by which most lncRNAs influence oncogenesis are unknown and require further investigation. We discuss several tumor-associated lncRNAs: H19, ANRIL, MALAT1, HOTAIR and MEG3.

The lncRNA H19, which is $2.3 \mathrm{~kb}$ in length and encoded by the maternally imprinted gene $H 19$ on chromosome 11p15.5, was the first IncRNA determined to be associated with cancer. H19 rapidly decreases in most tissues after birth, but is reactivated during carcinogenesis. H19 expression is elevated in several types of cancer, including hepatocellular, bladder and breast carcinomas, which suggests an oncogenic function [51]. The oncogene $c-M y c$ directly activates $\mathrm{H} 19$ by binding to the $H 19$ promoter [52], while the tumor suppressor p53 decreases H19 expression [48]. Either direct or indirect binding of the transcription factor E2F1 to the $H 19$ gene promoter stimulates cells to enter $\mathrm{S}$ phase and accelerates cell cycle progression, resulting in the proliferation of breast cancer cells [53]. In human colorectal cancer, miR-675 is processed from the first exon of $H 19$ and this miRNA inhibits the expression of the tumor suppressor gene retinoblastoma $(R B)$ to play an oncogenic role [54]. In contrast to its oncogenic effect, H19 appears to participate in tumor suppression in other contexts. Over-expression of H19 in two embryonic tumor cell lines, RD and G401, inhibits cell proliferation and tumorigenesis. In a mouse model of teratocarcinoma, embryos lacking H19 grew larger tumors than those expressing H19. In a similar model with hepatocarcinoma, mice developed tumor much earlier when H19 was absent [51].

ANRIL (antisense non-coding RNA in the INK4 locus) is an antisense lncRNA encoded by the INK4 locus of the INK4B/ARF/INK4A gene cluster on chromosome 9p21.3 and processed into multiple transcripts, including an unspliced transcript of $34.8 \mathrm{~kb}$ termed p15AS [55]. ANRIL is considered an oncogenic lncRNA due to its increased expression in leukemia and prostate cancer [36]. By regulating the expression of the tumor suppressor genes $p 15 / C D K N 2 B, p 16 / C D K N 2 A$ and $p 14 / A R F$ in the INK4B/ $A R F / I N K 4 A$ gene cluster, ANRIL modulates cell cycle progression and cellular senescence and subsequently promotes tumorigenesis [56]. ANRIL interacts with the CBX7 component of polycomb repressive complex 1 (PRC1) and recruits PRC1 to the INK4B/ARF/INK4A gene cluster, where PRC1 induces changes in the chromatin structure that silence the INK4B/ARF/INK4A gene cluster [36].

The lncRNA MALAT1 (metastasis-associated lung adenocarcinoma transcript 1), also known as NEAT2, is $7.5 \mathrm{~kb}$ in length and encoded by the MALATI gene located on chromosome 11q13.1. Cellular MALAT1 can be posttranscriptionally processed to yield the short, highly conserved, tRNA-like molecule mascRNA and the long MALAT1 transcript, which contains a poly(A) tail-like moiety [57]. MALAT1 is expressed in normal human tissues and exhibits increased expression in cancers of the breast, prostate, colon, liver, lung and others [58]. The expression level of MALAT1 in the metastatic tumors of non-small cell lung cancer (NSCLC) patients is three times higher than that of patients lacking metastatic tumors, and patients harboring tumors with elevated MALAT1 expression have a poor prognosis [59]. MALAT1 promotes lung adenocarcinoma cell migration in vitro by regulating cell motility-related genes at the transcriptional and/or posttranscriptional level [60]. The inhibition of MALAT1 reduces the proliferative and invasive potential of cervical cancer cells in vitro [61]. MALAT1 remains in the nucleus and localizes to nuclear speckles, where pre-mRNA processing occurs. A recent study revealed that MALAT1 regulates the alternative splicing of pre-mRNAs by modulating the activation of serine/arginine splicing factors [62]. This result implies that MALAT1 regulates the post-transcriptional processing or modification of RNA. MALAT1 binds to PSF (polypyrimidine tract binding protein-associated splicing factor) and prevents PSF from binding to the transcriptional regulatory region of the oncogene $C A G E 6$, which stimulates the abundant transcription of GAGE6 and thus promotes tumorigenesis [63].

HOTAIR (HOX antisense intergenic RNA), which is $2158 \mathrm{nt}$ in length, is an antisense lncRNA transcribed near the HOXC loci on chromosome 12q13.13 [37]. HOTAIR was discovered to be highly over-expressed in primary breast epithelial tumors and induced the invasion and metastasis of breast cancer [64]. The expression level of HOTAIR in hepatocellular carcinoma tissues was also higher than that of adjacent non-cancerous tissues, and the high levels of HOTAIR expression correlated with recurrence and a poor survival rate in liver cancer patients that received liver transplants [65]. In addition, decreasing the expression of HOTAIR in breast cancer and hepatocellular carcinoma cell lines inhibited tumor invasion [64,65]. Fur- 
thermore, inhibition of HOTAIR sensitized tumor cells to tumor necrosis factor $\alpha(\mathrm{TNF} \alpha)$-induced apoptosis, as well as apoptosis induced by treatment with chemotherapeutic agents cisplatin and doxorubicin [65]. HOTAIR functions as a molecular scaffold, binding at least two distinct histone modification complexes. The $5^{\prime}$ region of HOTAIR binds the PRC2 complex responsible for H3K27 methylation, and the $3^{\prime}$ region of HOTAIR binds the LSD1/CoREST/REST complex that mediates the demethylation of $\mathrm{H} 3 \mathrm{~K} 4$ [1]. HOTAIR recruits the PRC2 complex to specific target genes on a genome-wide scale, thereby facilitating abnormal H3K27 methylation and the epigenetic silencing of tumor metastasis suppressor genes, including JAM2, PCDH10, and $P C D H B 5$. Furthermore, the HOTAIR-mediated recruitment of PRC2 induces the expression of pro-metastasis genes, such as $A B L 2, S N A I L$, and Laminin, thereby promoting breast cancer metastasis [64].

MEG3 (maternally expressed gene 3), $1.6 \mathrm{~kb}$ in length, is encoded by the MEG3 gene of the DLK1/MEG3 locus on human chromosome $14 \mathrm{q} 32.3$. The $M E G 3$ gene is a maternally imprinted gene composed of 10 exons, and 12 MEG3 isoforms have been detected to date [66]. MEG3 is expressed in multi-organs and exhibits particularly high expression levels in the brain and the pituitary gland; however, MEG3 expression is reduced or absent in some tumors and tumor cell lines. Multiple mechanisms, including gene deletion, hypermethylation of the promoter and the intergenic region, contribute to the loss of MEG3 expression in tumors [67]. MEG3 inhibits the proliferation of tumor cells and modulates tumor angiogenesis by regulating p53, MDM2, $\mathrm{Rb}, \mathrm{p} 16^{\mathrm{INK} 4 \mathrm{~A}}$, Notch, VEGF and their signaling pathways, thereby suppressing tumorigenesis [68]. MDM2 is an important negative regulator of the $\mathrm{p} 53$ protein, and both $\mathrm{p} 53$ and MDM2 are target genes of MEG3 [69]. In tumor cells, MEG3 can either directly increase p53 expression or indirectly enhance $\mathrm{p} 53$ expression through targeted inhibition of MDM2 [69]. The activity of p53 requires the transcription of the full length MEG3 transcript, but not the translation of the MEG3 protein. Intriguingly, MEG3 fails to stimulate expression of the p53 target gene $p 21^{\text {Cipl }}$, but can increase the expression of the cell proliferation inhibitory gene GDF15 through p53 [69]. Further studies revealed that MEG3 enhances p53 binding to the GDF15 promoter but not to the $p 21^{\text {Cipl }}$ promoter [69]. Therefore, MEG3 activates p53 and selectively induces the expression of p53 target genes to inhibit the proliferation of tumor cells. Furthermore, MEG3-mediated functional activation of p53 depends on MEG3 secondary structure rather than the primary sequence of MEG3 [66]. MEG3 also suppresses tumor cell proliferation directly by regulating $\mathrm{Rb}$ phosphorylation and indirectly by activating the tumor suppressor gene $p 16^{I N K 4 A}$, which positively regulates $R b[68,70]$. In a $M e g 3$ knock-out mouse model, Meg3 was discovered to suppress angiogenesis by regulating VEGF signaling pathways [71]. Additionally, Meg3 regulates cell proliferation, differentiation and other important processes by modulating Notch signaling pathways to suppress tumorigenesis [71].

There are other IncRNAs that participate in carcinogenesis and cancer progression, including Zeb2 NAT (natural antisense transcripts, also known as antisense RNA), p21 NAT, CCND1 ncRNA, etc. The Zeb2 gene is a transcriptional repressor of $E$-cadherin. A recent study demonstrated that the NAT of Zeb2 (Zeb2 NAT, also known as Sip1 NAT) increases Zeb2 protein expression by regulating splicing and inhibiting E-cadherin expression in mesenchymal cells, which subsequently induces epithelial-to-mesenchymal transition (EMT) and promotes tumor metastasis [47]. The lncRNA p21 NAT, which is the NAT of the tumor suppressor $p 21 / C D K N 1 A$, cooperates with Ago-1 to induce histone $\mathrm{H} 3 \mathrm{~K} 27$ methylation of the $p 21 / C D K N 1 A$ promoter region, thereby inhibiting p21 expression and promoting tumorigenesis [72]. CCND1, encoding cyclin D1 protein, is an important cell cycle regulatory gene that, when over-expressed, induces malignant hyperplasia and promotes tumorigenesis. When cells are subjected to DNA damage, the lncRNA CCND1 ncRNA is transcribed from the regulatory sequence of the CCNDI gene promoter region [73]. The CCND1 ncRNA recruits the RNA-binding protein TLS (translocated in liposarcoma) to the CCND1 promoter region and converts the TLS protein from the inactive conformation into the active form. The modified TLS then binds to and inhibits CREB-binding protein (CBP) and p300 histone acetyltransferase activities, thereby reducing the expression of CCNDI [73] and suppressing tumorigenesis.

Many important biological functions of lncRNAs have been discovered recently as lncRNAs have become another hotspot in the field of molecular oncology. Substantial evidence indicates that IncRNAs participate in all steps of tumor initiation and development; therefore, thorough elucidation of their functions and molecular mechanisms are of great importance in the diagnosis and treatment of cancer. For example, the lncRNA DD3 demonstrated greater specificity than serum prostate-specific antigen (PSA) in predicting prostate tumors and is being developed into a new diagnostic marker for prostate cancer [74]. HOTAIR may serve as a potential biomarker for the lymph node metastasis of hepatocellular carcinoma [65]. Additionally, preventing the interaction between HOTAIR and the PRC2 or LSD1 complex may limit the metastatic potential of breast cancer cells [48]. Although our current knowledge on lncRNAs is only the tip of the iceberg, novel methods and technology will eventually lift the mysterious veil covering lncRNAs, thereby enabling the development of new cancer therapeutic strategies.

This work was supported by the National Natural Science Foundation of China (Grant Nos. 30971147, 81071644, 81172189, 81171930, 81272255, 81272297, 81272298 and 91229122), the Hunan Province Natural Science Foundation of China (Grant No. 10JJ7003), the Fok Ying Tong Education 
Foundation (Grant No. 121036), the Fundamental Research Funds for the Central Universities (Grant No. 2011JQ020), the Mittal Innovative Entrepreneurial Project of Central South University (Grant No. 11MX27), the Open-End Fund for the Valuable and Precision Instruments of Central South University and the Postdoctoral Science Foundation of Central South University.

1 Tsai M C, Manor O, Wan Y, et al. Long noncoding RNA as modular scaffold of histone modification complexes. Science, 2010, 329: 689-693

2 Ponting C P, Oliver P L, Reik W. Evolution and functions of long noncoding RNAs. Cell, 2009, 136: 629-641

3 Wilusz J E, Sunwoo H, Spector D L. Long noncoding RNAs: functional surprises from the RNA world. Genes Dev, 2009, 23: 1494-1504

4 Huang W T, Guo X Q, Dai J P, et al. MicroRNA and lncRNA in neurodegenerative diseases. Prog Biochem Biophys, 2010, 37: 826-833

5 Yang $\mathrm{C}$, Wei W. The miRNA expression profile of the uveal melanoma. Sci China Life Sci, 2011, 54: 351-358

6 Feng Y, Yu X. Cardinal roles of miRNA in cardiac development and disease. Sci China Life Sci, 2012, 54: 1113-1120

7 Lin B B, Zhang Y, Xu Y, et al. The main miRNA regulators Drosha and Dicer are associated with ectopic endometrium. Prog Biochem Biophys, 2012, 39: 78-85

8 Liang J W, Wang $\mathrm{P}$, Chen $\mathrm{L}$, et al. miR-133b may regulate mouse B cell development by targeting the transcription factor foxO1. Prog Biochem Biophys, 2011, 38: 744-750

9 Chen H, Chen Q, Fang M, et al. microRNA-181b targets MLK2 in HL-60 cells. Sci China Life Sci, 2010, 53: 101-106

10 Luo J, Teng M, Fan J, et al. Marek's disease virus-encoded microRNAs: genomics, expression and function. Sci China Life Sci, 2010, 53: 1174-1180

11 Yan L M, Wu J Y, Yu X H, et al. Role of intronic microRNA in the regulation of endothelial nitric oxide synthase expression and the proliferation of endothelial cells. Prog Biochem Biophys, 2010, 37: 747-753

12 Chen W J, Yin K, Zhao G J, et al. microRNAs: a new mechanisms for regulation of lipid metabolism. Prog Biochem Biophys, 2011, 38: 781-790

13 Ding Y F, Zhu C, Wang S S, et al. Regulation of heavy metal stress response by plant microRNAs. Prog Biochem Biophys, 2011, 38: 1106-1110

14 Jin Y F, Xu G M, Li Y, et al. A novel normalization approach for microRNA quantitative PCR. Prog Biochem Biophys, 2011, 38: 473-481

15 Li S, Yu B, Wang Y, et al. Identification and functional annotation of novel microRNAs in the proximal sciatic nerve after sciatic nerve transection. Sci China Life Sci, 2011, 54: 806-812

16 Wang G, Dong X, Hu J, et al. Long-term ex vivo monitoring of in vivo microRNA activity in liver using a secreted luciferase sensor. Sci China Life Sci, 2011, 54: 418-425

17 Zuo J, Wang Y, Liu H, et al. MicroRNAs in tomato plants. Sci China Life Sci, 2011, 54: 599-605

18 Feng W, Feng Y. MicroRNAs in neural cell development and brain diseases. Sci China Life Sci, 2012, 54: 1103-1112

19 Gong Z J, Huang H B, Xu K, et al. Advances in microRNAs and TP53 gene regulatory network. Prog Biochem Biophys, 2012, 39:1133-1144

20 Li J, Wei H, Li Y, et al. Identification of a suitable endogenous control gene in porcine blastocysts for use in quantitative PCR analysis of microRNAs. Sci China Life Sci, 2012, 55: 126-131

21 Yu J, Wang F. Recent progress in microRNA study: benefits from technique advance. Sci China Life Sci, 2012, 55: 649-650

22 Zhang Y. Progress, challenges and new concepts in microRNAs. Sci China Life Sci, 2012, 54: 1096

23 Zhang Y, Dong D, Yang B. Atrial remodeling in atrial fibrillation and association between microRNA network and atrial fibrillation. Sci
China Life Sci, 2012, 54: 1097-1102

24 Ye L, Zhang G Y, Liu T, et al. Construction of combination gene vector expressing VEGF-siRNA and fusion suicide gene yCDglyTK and its application. Prog Biochem Biophys, 2010, 37: 503-509

25 Zhang Y, Zu X Y, Luo W S, et al. siRNA induced cyclinB1 knockdown sensitizes HepG2 cells to daunorubicin. Prog Biochem Biophys, 2011, 38: 551-557

26 Chen R S. Delivery: a principal challenge in siRNA application. Prog Biochem Biophys, 2012, 39: 395

27 Dong W J, Zhou Y J, Liang W. Lipid-based siRNA delivery systems. Prog Biochem Biophys, 2012, 39: 396-401

28 Wang $\mathrm{H} \mathrm{J}$, Yin J Q. The effects of cell penetrating peptides structure modification on their siRNA delivery function in vitro and in vivo. Prog Biochem Biophys, 2012, 39: 402-409

29 Jiang L, Wei C, Li Y. Viral suppression of RNA silencing. Sci China Life Sci, 2012, 55: 109-118

30 Tan Y, Zhang X M. Aptamer mediated delivery of small interfering RNAs. Prog Biochem Biophys, 2012, 39: 410-415

31 Zhu $\mathrm{H}$, Guo $\mathrm{H}$. The role of virus-derived small interfering RNAs in RNA silencing in plants. Sci China Life Sci, 2012, 55: 119-125

32 Zheng J, Zheng B J, Fang F, et al. Mitochondrial tRNA mutations associated with hearing loss. Prog Biochem Biophys, 2012, 39: 22-30

33 Qi G. The background of the total synthesis of yeast alanine transfer RNA. Sci China Life Sci, 2010, 53: 19-21

34 Chen X, Wang E D. Transfer RNA-derived Small RNAs: degradation fragments or novel regulatory molecules? Prog Biochem Biophys, 2011, 38: 681-687

35 Li X, Wu Z, Fu X, et al. Long noncoding RNAs: insights from biological features and functions to diseases. Med Res Rev, 2012

36 Yap K L, Li S, Munoz-Cabello A M, et al. Molecular interplay of the noncoding RNA ANRIL and methylated histone H3 lysine 27 by polycomb CBX7 in transcriptional silencing of INK4a. Mol Cell, 2010, 38: 662-674

37 Rinn J L, Kertesz M, Wang J K, et al. Functional demarcation of active and silent chromatin domains in human HOX loci by noncoding RNAs. Cell, 2007, 129: 1311-1323

38 Liang X, Ma J, Schatten H, et al. Epigenetic changes associated with oocyte aging. Sci China Life Sci, 2012, 55: 670-676

39 Huang H, Jiao R. Roles of chromatin assembly factor 1 in the epigenetic control of chromatin plasticity. Sci China Life Sci, 2012, 55: $15-19$

40 Chao S, Li J, Jin X, et al. Epigenetic reprogramming of embryos derived from sperm frozen at -20 degrees C. Sci China Life Sci, 2012, 55: 349-357

41 Bi X. Functions of chromatin remodeling factors in heterochromatin formation and maintenance. Sci China Life Sci, 2012, 55: 89-96

42 Ling $\mathrm{H} \mathrm{Y,} \mathrm{Hu} \mathrm{B,} \mathrm{Feng} \mathrm{S} \mathrm{D,} \mathrm{et} \mathrm{al.} \mathrm{The} \mathrm{role} \mathrm{of} \mathrm{epigenetic} \mathrm{regulation} \mathrm{in}$ diabetes and its complications. Prog Biochem Biophys, 2012, 39: $14-21$

43 Imamura T, Yamamoto S, Ohgane J, et al. Non-coding RNA directed DNA demethylation of Sphk1 CpG island. Biochem Biophys Res Commun, 2004, 322: 593-600

44 Martens J A, Laprade L, Winston F. Intergenic transcription is required to repress the Saccharomyces cerevisiae SER3 gene. Nature, 2004, 429: 571-574

45 Martianov I, Ramadass A, Serra Barros A, et al. Repression of the human dihydrofolate reductase gene by a non-coding interfering transcript. Nature, 2007, 445: 666-670

46 Mariner P D, Walters R D, Espinoza C A, et al. Human Alu RNA is a modular transacting repressor of mRNA transcription during heat shock. Mol Cell, 2008, 29: 499-509

47 Beltran M, Puig I, Pena C, et al. A natural antisense transcript regulates Zeb2/Sip1 gene expression during Snaill-induced epithelial-mesenchymal transition. Genes Dev, 2008, 22: 756-769

48 Gibb E A, Brown C J, Lam W L. The functional role of long non-coding RNA in human carcinomas. Mol Cancer, 2011, 10: 38

49 Yang F, Zhang L, Huo X S, et al. Long noncoding RNA high expression in hepatocellular carcinoma facilitates tumor growth 
through enhancer of zeste homolog 2 in humans. Hepatology, 2011, 54: 1679-1689

50 Khaitan D, Dinger M E, Mazar J, et al. The melanoma-upregulated long noncoding RNA SPRY4-IT1 modulates apoptosis and invasion. Cancer Res, 2011, 71: 3852-3862

51 Matouk I J, DeGroot N, Mezan S, et al. The H19 non-coding RNA is essential for human tumor growth. PLoS One, 2007, 2: e845

52 Barsyte-Lovejoy D, Lau S K, Boutros P C, et al. The c-Myc oncogene directly induces the H19 noncoding RNA by allele-specific binding to potentiate tumorigenesis. Cancer Res, 2006, 66: 5330-5337

53 Berteaux N, Lottin S, Monte D, et al. H19 mRNA-like noncoding RNA promotes breast cancer cell proliferation through positive control by E2F1. J Biol Chem, 2005, 280: 29625-29636

54 Tsang W P, Ng E K, Ng S S, et al. Oncofetal H19-derived miR-675 regulates tumor suppressor $\mathrm{RB}$ in human colorectal cancer. Carcinogenesis, 2010, 31: 350-358

$55 \mathrm{Yu} \mathrm{W}$, Gius D, Onyango P, et al. Epigenetic silencing of tumour suppressor gene p15 by its antisense RNA. Nature, 2008, 451: 202-206

56 Pasmant E, Sabbagh A, Vidaud M, et al. ANRIL, a long, noncoding RNA, is an unexpected major hotspot in GWAS. FASEB J, 2011, 25: $444-448$

57 Wilusz J E, Freier S M, Spector D L. 3' end processing of a long nuclear-retained noncoding RNA yields a tRNA-like cytoplasmic RNA. Cell, 2008, 135: 919-932

58 Perez D S, Hoage T R, Pritchett J R, et al. Long, abundantly expressed non-coding transcripts are altered in cancer. Hum Mol Genet, 2008, 17: 642-655

59 Ji P, Diederichs S, Wang W, et al. MALAT-1, a novel noncoding RNA, and thymosin beta4 predict metastasis and survival in early-stage non-small cell lung cancer. Oncogene, 2003, 22: 8031-8041

60 Tano K, Mizuno R, Okada T, et al. MALAT-1 enhances cell motility of lung adenocarcinoma cells by influencing the expression of motility-related genes. FEBS Lett, 2010, 584: 4575-4580

61 Guo F, Li Y, Liu Y, et al. Inhibition of metastasis-associated lung adenocarcinoma transcript 1 in CaSki human cervical cancer cells suppresses cell proliferation and invasion. Acta Biochim Biophys Sin,
2010, 42: 224-229

62 Tripathi V, Ellis J D, Shen Z, et al. The nuclear-retained noncoding RNA MALAT1 regulates alternative splicing by modulating SR splicing factor phosphorylation. Mol Cell, 2010, 39: 925-938

63 Li L, Feng T, Lian Y, et al. Role of human noncoding RNAs in the control of tumorigenesis. Proc Natl Acad Sci USA, 2009, 106: 12956-12961

64 Gupta R A, Shah N, Wang K C, et al. Long non-coding RNA HOTAIR reprograms chromatin state to promote cancer metastasis. Nature, 2010, 464: 1071-1076

65 Yang Z, Zhou L, Wu L M, et al. Overexpression of long non-coding RNA HOTAIR predicts tumor recurrence in hepatocellular carcinoma patients following liver transplantation. Ann Surg Oncol, 2011, 18: $1243-1250$

66 Zhang X, Rice K, Wang Y, et al. Maternally expressed gene 3 (MEG3) noncoding ribonucleic acid: isoform structure, expression, and functions. Endocrinology, 2010, 151: 939-947

67 Zhou Y, Zhang X, Klibanski A. MEG3 noncoding RNA: a tumor suppressor. J Mol Endocrinol, 2012, 48: R45-53

68 Benetatos L, Vartholomatos G, Hatzimichael E. MEG3 imprinted gene contribution in tumorigenesis. Int J Cancer, 2011, 129: 773-779

69 Zhou Y, Zhong Y, Wang Y, et al. Activation of p53 by MEG3 non-coding RNA. J Biol Chem, 2007, 282: 24731-24742

70 Miao J W, Zhang Y Q, Xu C Y, et al. Involvement of P16(INK4a) and sonic hedgehog signaling pathways in squamous cell carcinoma of uterine cervix and its precursor lesions. Prog Biochem Biophys, 2012, 39: 671-677

71 Gordon F E, Nutt C L, Cheunsuchon P, et al. Increased expression of angiogenic genes in the brains of mouse meg3-null embryos. Endocrinology, 2010, 151: 2443-2452

72 Morris K V, Santoso S, Turner A M, et al. Bidirectional transcription directs both transcriptional gene activation and suppression in human cells. PLoS Genet, 2008, 4: e1000258

73 Wang X, Arai S, Song X, et al. Induced ncRNAs allosterically modify RNA-binding proteins in cis to inhibit transcription. Nature, 2008, 454: 126-130

74 Tinzl M, Marberger M, Horvath S, et al. DD3PCA3 RNA analysis in urine-a new perspective for detecting prostate cancer. Eur Urol, 2004, 46: 182-186

Open Access This article is distributed under the terms of the Creative Commons Attribution License which permits any use, distribution, and reproduction in any medium, provided the original author(s) and source are credited. 